\title{
Predictive value of platelet aggregation rate in postpartum deep venous thrombosis and its possible mechanism
}

\author{
MINGXIA SUN, CHONGDONG LIU, NA ZHAO, KAIKAI MENG and ZHENYU ZHANG \\ Department of Obstetrics and Gynecology, Beijing Chaoyang Hospital, Beijing 100043, P.R. China
}

Received September 15, 2017; Accepted February 26, 2018

DOI: 10.3892/etm.2018.6116

\begin{abstract}
The present study investigated the predictive value of the platelet aggregation rate in postpartum deep venous thrombosis and its possible mechanism. From January 2014 to January 2016, 23 patients with postpartum deep vein thrombosis of lower extremity treated in the Department of Obstetrics of Beijing Chaoyang Hospital were as assigned as the observation group. At the same time, 25 cases with normal recovery were assigned as the control group. Blood samples were collected from all the subjects. The platelet aggregation rate was measured using a platelet aggregation apparatus. Plasma platelet activating factor (PAF) levels were measured by ELISA. The positive rate of platelet P-selectin (CD62p) and lysosomal membrane glycoprotein (CD63) was measured by flow cytometry. PI3K expression and AKt phosphorylation levels were measured by western blot analysis. The ROC curve was used to evaluate the value of the platelet aggregation rate in predicting postpartum deep vein thrombosis of lower extremity. The correlation between the platelet aggregation rate and PAF and PI3K/AKt expression was also analyzed. The cesarean section rate, platelet 5-min maximum aggregation rate, PAF level and the positive rate of CD62p and CD63 were significantly higher in the control than those in the observation group $(\mathrm{P}<0.05)$. Furthermore, the platelet aggregation rate was positively correlated with the expression of PAF, CD62p and CD63 ( $\mathrm{r}=0.389,0.451$, and 0.452; all $\mathrm{P}<0.05$ ). The platelet 5-min maximum aggregation rate for predicting postpartum deep vein thrombosis of lower extremity was reflected by the area under the ROC curve (AUC $=0.797$, $\mathrm{P}=0.000)$. The $\mathrm{PI} 3 \mathrm{Kp} 110 \beta / \beta$-actin and $\mathrm{p}$-AKt/AKt ratio was significantly higher in the observation compared with the control group $(\mathrm{P}<0.05)$. In addition, the platelet aggregation rate was positively correlated with the expression of $\mathrm{PI} 3 \mathrm{~K}$ and phosphorylation level of AKt $(\mathrm{r}=0.441,0.430$; all $\mathrm{P}<0.05)$. The
\end{abstract}

Correspondence to: Dr Mingxia Sun, Department of Obstetrics and Gynecology, Beijing Chaoyang Hospital, 5 Jingyuan Road, Shijingshan, Beijing 100043, P.R. China

E-mail: smx503@163.com

Key words: deep vein thrombosis, platelet aggregation, $\mathrm{PI} 3 \mathrm{~K} / \mathrm{AKt}$ signaling pathway results suggested that platelet aggregation activity is elevated in postpartum deep vein thrombosis patients. It has a certain predictive value for the occurrence of postpartum deep vein thrombosis of lower extremity. Thuss, the PI3K/AKt signaling pathway may be one of the mechanisms of platelet aggregation.

\section{Introduction}

Deep vein thrombosis is one of the common complications of postpartum (1). Maternal blood composition, exacerbated hypercoagulable state of the blood caused by the changes in blood cell activity, pregnancy uterine oppression, slow deep vein blood flow caused by postpartum bed rest and breastfeeding while sitting, combined with birth trauma activation of coagulation, has made women a high-risk population for deep venous thrombosis. Deep vein thrombosis has high morbidity and mortality, and is a serious threat to maternal life and health (2).

Early identification and active intervention can effectively reduce the incidence and mortality rates of deep vein thrombosis. However, clinical methods for detecting deep vein thrombosis cannot effectively identify the early changes of deep vein thrombosis. Therefore, strengthening studies on the development of laws and the pathogenesis of deep vein thrombosis would contribute to the early identification of deep vein thrombosis of lower extremity. Platelet aggregation is an important part of thrombus formation (3). A related study revealed that (4) the PI3K/AKt signaling pathway can be involved in the regulation of platelet adhesion and aggregation activity (5). However, changes in platelet aggregation rate and the PI3K/AKt signaling pathway in postpartum deep venous thrombosis patients needs to be further confirmed.

In the present study, platelet aggregation rate, platelet activity indicators and PI3K expression level, and changes in $\mathrm{AKt}$ phosphorylation were preliminarily analyzed in postpartum deep vein thrombosis patients, in order to explore the platelet aggregation activity changes and its possible mechanism in postpartum deep vein thrombosis patients, with the aim to provide a theoretical basis for the early diagnosis of patients with postpartum deep vein thrombosis of lower extremity.

\section{Materials and methods}

Patients. A total of 23 patients with postpartum deep vein thrombosis of lower extremity treated in the Department of 
Obstetrics of Beijing Chaoyang Hospital (Beijing, China) from January 2014 to January 2016 were selected for the study. The age of these patients was 21-39 years, and the mean age was $28.75 \pm 6.72$ years. Of the 23 patients, 14 patients had cesarean section, while 9 patients had vaginal delivery. These patients were assigned as the observation group. The inclusion criteria were as follows: i) Lower extremity swelling, pain, superficial varicose veins and other clinical manifestations for deep vein thrombosis; and ii) deep venous thrombosis diagnosed by ultrasound. During the same period, 25 patients with normal recovery were assigned as the control group. The age of these patients was 21-40 years, and the mean age was 29.29 \pm 8.01 years. Of these patients, eight patients had cesarean section, while 17 patients had vaginal delivery. The inclusion criteria were as follows: i) No clinical manifestations for deep venous thrombosis, and no history of thrombosis and ii) no deep venous thrombosis detected by ultrasound. Exclusion criteria for the two groups were: i) Patients who had pregnancy-induced hypertension, gestational glucose, placental abruption and other pregnancy disorders; ii) patients with severe heart, liver and kidney dysfunction, or severe infection and iii) patients associated with immune system diseases or active bleeding and other diseases. In the present study, all the subjects or their families provided signed informed consent. This study was approved by the Ethics Committee of Beijing Chaoyang Hospital.

\section{Methods}

Groupings. Patients were diagnosed for postpartum deep venous thrombosis according to the diagnostic and therapeutic criteria for deep venous thrombosis (2015 revision) (6) were assigned as the observation group. During the same period, 25 postpartum with normal recovery were assigned as the control group.

Collection and processing of blood samples. Since deep venous thrombosis occurred within 1-2 weeks postpartum (7), venous blood samples at two weeks postpartum in the control group and venous blood samples after diagnosis in the observation group were tested. The collected venous blood was centrifuged for $30 \mathrm{~min}$ at $1,500 \mathrm{x} \mathrm{g}$ at $4^{\circ} \mathrm{C}$ the plasma was drawn, and GPIb and platelet-activating factor (PAF) content was determined. The specimens were centrifuged for $10 \mathrm{~min}$ at $700 \mathrm{xg}$ at $4^{\circ} \mathrm{C}$ to obtain the upper plasma, the platelet rich plasma (PRP) was prepared, centrifuged again for $15 \mathrm{~min}$ at $700 \mathrm{xg}$ at $4^{\circ} \mathrm{C}$ to draw the upper plasma, the platelet poor plasma (PPP) was prepared and the number of platelets were measured in five categories using a hemocytometer (Abbott Pharmaceutical Co. Ltd., Lake Bluff, IL, USA). PPP was adjusted with PRP to a platelet concentration of $3 \times 10^{8}$ cells $/ \mathrm{ml}$ was counted, and these were stored by cryopreservation.

Determination of platelet aggregation rate. The adjusted PRP was placed into a four-channel platelet aggregation instrument (Bio-Rad Laboratories, Inc., Hercules, CA, USA) to determine the 5-min maximum platelet aggregation rate with ADP as an inducer (at a final concentration of $20 \mu \mathrm{mol} / \mathrm{l}$ ).

Detection of platelet activity indicators. The blood GPIb and PAF content of subjects were determined using an ELISA kit purchased from a test company (R\&D Systems, Minneapolis, MN, USA), and the test procedure was carried out in strict accordance with reagent instructions. Flow cytometry was used to measure platelet membrane platelet P-selectin (CD62p) and lysosomal membrane glycoprotein (CD63) positive rate. After PRP was adjusted, 1\% paraformaldehyde was added, fixed in room temperature for $15 \mathrm{~min}$, washed twice with PBS-EDTA solution, centrifuged, and the supernatant was taken. Then, CD62p-FITC and CD63-FITC, were added, mixed by blowing, allowed to react for $30 \mathrm{~min}$ in the dark at room temperature, washed twice, and suspended in PBS solution. Then, flow cytometry (Bio-Rad Laboratories, Inc., Hercules, CA, USA) was performed for detection. An excitation wavelength of $490 \mathrm{~nm}$ was selected, the angular voltage was adjusted, the target platelet community was divided, and the positive rate of CD62p and CD63 was calculated.

Detection of PI3K and AKt phosphorylation levels. The adjusted PRP was added to the lysate to lyse the platelets, the protein concentration was measured using the BCA method, the sample was run on the gel, transferred onto a membrane, closed with milk, and incubated with primary rabbit anti-human AKt polyclonal antibody (1:600; cat. no. LS-C183748-100), phosphorylated AKt polyclonal antibody (1:1,000; cat. no. LS-C122634-50), PI3Kp110 $\beta$ monoclonal antibody (1:1,000; cat. no. 3011) and $\beta$-actin monoclonal antibody (1:1,000; cat. no. LS-B1625). Then it was incubated with goat anti-rabbit secondary polyclonal antibody (1:1500; cat. no. LS-C56309). All antibodies were purchased from LifeSpan BioSciences, Inc. (Seattle, WA, USA). The PI3Kp110 $\beta$ antibody was purchased from Cell Signaling Technology, Inc. (Danvers, MA, USA). Then, an ECL luminescent developer was added, exposed and imaged.

Statistical analysis. SPSS 19.0 (SPSS, Inc., Chicago, IL, USA) software was used to analyze the experimental data. The platelet aggregation rate, platelet activity indicators and other measurement data were presented as mean \pm standard deviation (SD). The t-test was used to compare the mean of two groups, and countable data by Chi-square test were performed. The ROC curve was drawn to evaluate the value of platelet aggregation in predicting the occurrence of postpartum deep venous thrombosis of lower extremity. Pearson's correlation was performed to analyze the platelet aggregation rate and the plasma platelet activity factor content of subjects, and a correlation analysis was performed on the expression level of PI3K/AKt. P<0.05 was considered to indicate a statistically significant difference.

\section{Results}

Comparison of the general information and platelet aggregation rate between the two groups. A comparison of the age and 5-min maximum platelet aggregation rate between the two groups is shown in Table I. The difference in age between the two groups was not statistically significant $(\mathrm{P}>0.05)$. However, compared with the control group, the cesarean section rate and 5 -min maximum platelet aggregation rate of subjects in the observation group was significantly increased $(\mathrm{P}<0.05)$. the occurrence of postpartum deep venous thrombosis of 
Table I. Comparison of general data and the platelet aggregation rate between the two groups.

\begin{tabular}{lcccc}
\hline Groups & No. of cases & Age (years) & $\begin{array}{c}\text { Cesarean section } \\
\text { rate }(\%)\end{array}$ & $\begin{array}{c}\text { Five-minute maximum } \\
\text { platelet aggregation rate }(\%)\end{array}$ \\
\hline Observation & 23 & $28.75 \pm 6.72$ & 60.87 & $62.57 \pm 11.63$ \\
Control & 25 & $29.29 \pm 8.01$ & 20.00 & $49.06 \pm 10.74^{\mathrm{a}}$ \\
t-test $/ \chi^{2}$ & & -0.252 & 8.367 & 4.184 \\
P-value & 0.802 & 0.004 & $>0.05$ \\
\hline
\end{tabular}

${ }^{a}$ Compared with the observation group, $\mathrm{P}<0.05$.

Table II. Comparison of platelet activity indicators between the two groups.

\begin{tabular}{lcccc}
\hline Groups & No. of cases & PAF $(\mathrm{pg} / \mathrm{ml})$ & CD62p $(\%)$ & CD63 (\%) \\
\hline Observation & 23 & $13.40 \pm 2.34$ & $31.07 \pm 6.37$ & $36.00 \pm 9.63$ \\
Control & 25 & $8.55 \pm 1.56^{\mathrm{a}}$ & $14.51 \pm 3.54^{\mathrm{a}}$ & $19.71 \pm 4.06^{\mathrm{a}}$ \\
t-test & & 8.513 & 11.253 & 7.748 \\
P-value & $<0.05$ & $<0.05$ & $<0.05$
\end{tabular}

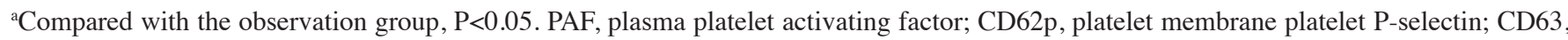
lysosomal membrane glycoprotein.

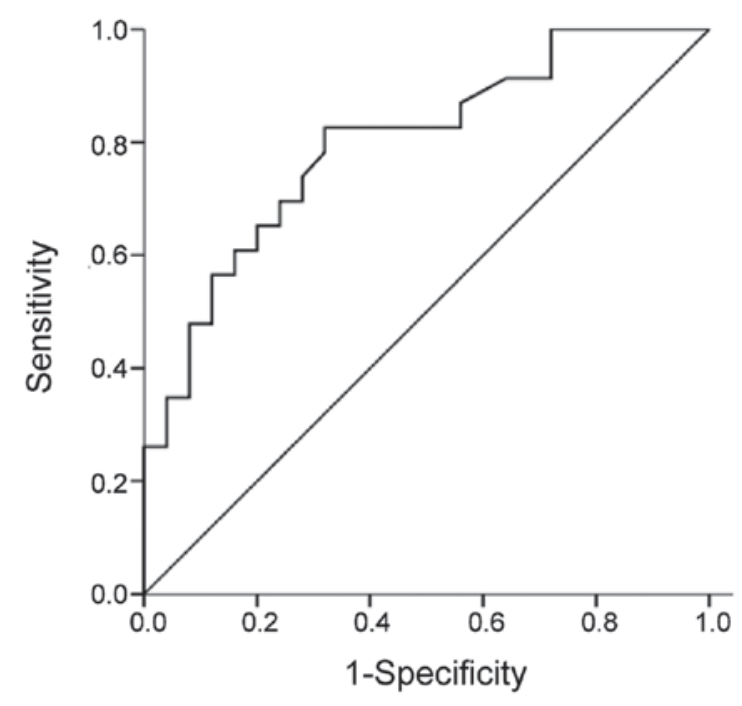

Figure 1. ROC curve of the platelet aggregation rate for predicting postpartum deep vein thrombosis of lower extremity.

lower extremity. Postpartum deep vein thrombosis patients with illness in the observation group $=1$ and normal recovery subjects without illness in the control group $=0$, and the ROC curve of the 5-min maximum platelet aggregation rate to predict the occurrence of postpartum deep vein thrombosis was drawn (Fig. 1). The area under the ROC curve (AUC) was 0.797 ( $\mathrm{P}>0.05,95 \%$ CI: 0.672-0.923).

Analysis of the platelet activity indicators of subjects and its correlation with platelet aggregation rate. The expression of PAF, CD62p and CD63 was detected in subjects in the two groups, and the results were analyzed. The expression levels of
Table III. The correlation between platelet activity indicators and the platelet aggregation rate.

\begin{tabular}{llll}
\hline $\begin{array}{l}\text { Correlation } \\
\text { index }\end{array}$ & PAF & CD62p & CD63 \\
\hline r-value & 0.389 & 0.451 & 0.452 \\
P-value & 0.006 & 0.001 & 0.001 \\
\hline
\end{tabular}

the three platelet activity indicators were significantly higher in the observation than those in the control group, (all $\mathrm{P}<0.05$, Table II). The correlation between the platelet activity indicators and platelet aggregation rate in subjects was analyzed, and it was found that the expression levels of PAF, CD62p and CD63 were positively correlated with the platelet aggregation rate $(\mathrm{r}=0.389,0.451$ and 0.452 ; all $\mathrm{P}<0.05$ (Table III).

Change of PI3K and AKt expression in platelets. Change of $\mathrm{PI} 3 \mathrm{~K}$ and AKt expression in platelets of subjects in the two groups were detected by western blot analysis to analyze the gray value of each band. In the observation group, the PI3Kp110 $/ / \beta$-actin and p-AKt/AKt gray value ratios of subjects were significantly higher than those in the control group (all $\mathrm{P}<0.05$; Fig. 2).

Analysis of the correlation between the platelet aggregation rate of subjects and the change of PISK and AKt expression in platelets. The correlation between the platelet aggregation rate of subjects and PI3K expression and AKt phosphorylation levels in platelets were analyzed. Results revealed that the platelet aggregation rate of subjects was positively correlated 
A

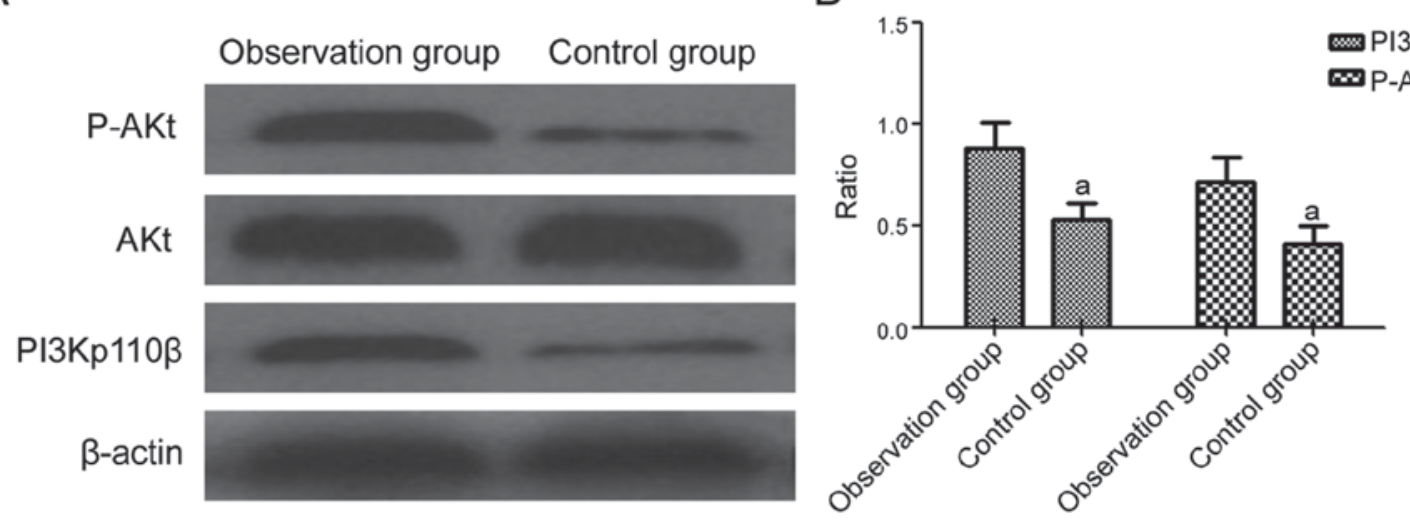

Figure 2. Change of PI3K and AKt expression in platelets. ${ }^{\mathrm{a}} \mathrm{P}<0.05$, compared with observation group.


Figure 3. Correlation between the platelet aggregation rate and the PI3K expression and AKt phosphorylation levels in platelets.

with PI3K levels and p-AKt levels in platelets $(\mathrm{r}=0.441,0.430$; all $\mathrm{P}<0.05$; Fig. 3).

\section{Discussion}

Due to changes in maternal blood physiology and anatomical structures, and the two risk factors of slow deep venous blood flow and blood hypercoagulable state, postpartum deep venous thrombosis has become a common postpartum complication (8). Strengthening the study of the mechanism of deep venous thrombosis and determining effective diagnostic indicators would contribute to the early prevention and treatment of postpartum deep vein thrombosis. Platelets are important components of thrombosis. They play a vital role in the formation of thrombus, and activate platelet adhesion, aggregation and the release of active substances that participate in the series of changes in thrombosis formation. Hence, the platelet aggregation rate is increased in patients with thrombosis, and the expression of platelet-associated active substances is enhanced. In addition, findings have shown that the expression of the PI3K/AKt pathway in platelets was enhanced in thrombosis patients (9), the PI3K/AKt pathway participates in cell activation, proliferation, apoptosis and other processes (10), and platelet adhesion, aggregation and release are closely related (11). Therefore, it could be considered that the activation of the PI3K/AKt signaling pathway is one of the mechanisms of platelet activation. The changes in platelet activity and the PI3K/AKt signaling pathway in patients with postpartum deep vein thrombosis remain to be confirmed. Therefore, in the present study, 23 subjects with postpartum deep venous thrombosis were assigned to the observation group and the 25 subjects with postpartum normal recovery were assigned to the control group. The platelet aggregation rate, PAF, CD62p, CD63, as well as the platelet activity indicators and expression level of the PI3K/AKt pathway, were analyzed to investigate platelet activity changes in postpartum deep vein thrombosis patients, and the mechanism involved.

Platelet adhesion aggregation is a key link in thrombosis. Adhesion molecules in plasma bind to platelet surface glycoprotein receptors to mediate platelet aggregation, blood platelet aggregation substance expression in thrombus patients is enhanced, platelets are activated under the action of a variety of coagulation factors, the amount of platelet membrane glycoprotein and plasma adhesive molecule expression becomes significantly elevated, and a large number of procoagulant platelet factors are released into the blood. Therefore, thrombosis may promote the expression 
of PAF (12) and significantly increase the platelet release of CD62p (13), CD63 and other (14) platelet activity markers. Thus, the platelet aggregation rate of thrombosis patients is increased. In the study conducted by Signorelli et al, it was suggested that the platelet aggregation activity of patients with deep vein thrombosis of lower extremity is enhanced (15). By analyzing the expression of the three substances (PAF, CD62p, and CD63) in the two groups, it was found that the expression levels of the three substances were significantly higher in postpartum deep vein thrombosis patients than in subjects with normal recovery. Similar results were obtained in studies conducted by Gerdsen et al (16) and Malaponte et al (17). The analysis of the platelet aggregation rate revealed that the 5-min maximum platelet aggregation rate for postpartum deep vein thrombosis of lower extremity was significantly higher than that for postpartum normal recovery. Furthermore, the expression level of the three substances was positively correlated with the platelet aggregation rate, suggesting that platelet aggregation activity increased in postpartum deep vein thrombosis patients. The ROC curve of postpartum deep venous thrombosis was predicted through the 5-min maximum platelets aggregation. The AUC obtained was 0.797 ( $\mathrm{P}>0.05)$, and it was observed that the increased platelet aggregation rate has a certain value in predicting postpartum deep venous thrombosis. These results suggest that the platelet aggregation activity of postpartum deep venous thrombosis of lower extremity is significantly enhanced, and has a certain predictive value for the occurrence of the disease.

The PI3K/AKt pathway intervenes with the platelet adhesion process by mediating the GPIb-IX signal (18). PI3K can affect the activity of GPIb, GPIIb/IIIa and GPIV (19), which plays a role in controlling platelet aggregation and release; while $\mathrm{AKt}$ platelets could promote the release of the active substance (20). The study conducted by Chen et al revealed that the tryptophan derivative CD-26 exerts an anti-thrombotic effect by inhibiting the PI3K/Akt pathway in platelets (9). Furthermore, the study conducted by Hao et al suggested that lutein A may inhibit platelet activation by inhibiting the PI3K/Akt pathway (21). Thus, the PI3K/AKt pathway is an important participant in the regulation of platelet activity. Western blot analysis was used in this study to detect the PI3Kp110 $\beta$ and p-AKt levels of platelets in subjects. The analysis revealed that PI3Kp110 $\beta$ expression and AKt phosphorylation levels were significantly higher in postpartum deep vein thrombosis patients than in subjects in the control group, suggesting that the expression of the PI3K/AKt pathway in postpartum deep vein thrombosis patients was enhanced. A correlation analysis on PI3Kp110 $\beta$ expression and AKt phosphorylation levels and the platelet aggregation rates of subjects was conducted. It was found that the platelet aggregation rate was positively correlated with PI3Kp110 $\beta$ expression and AKt phosphorylation levels. The findings suggests, that the enhancement of the PI3K/AKt signaling pathway in postpartum deep vein thrombosis patients may be one of the mechanisms of platelet aggregation.

Platelet is a key link in thrombosis. The study of changes in platelet function and its mechanism for patients with thrombosis would help to determine the early diagnostic indicators of thrombosis, providing a theoretical basis for the early prevention and treatment of thrombosis. Due to the limited amount of experimental samples, the changes in platelet activation and aggregation activity, and the expression level of the intracellular PI3K/AKt signaling pathway in patients with postpartum deep vein thrombosis remain to be further studied. It can only be speculated that the enhancement of the expression of the PI3K/AKt pathway is one of the mechanisms of platelet aggregation in postpartum deep vein thrombosis patients. The intracellular signal transduction process is very complex. Therefore, the specific mechanism of platelet aggregation activity in patients with postpartum deep vein thrombosis needs to be further confirmed. It is considered that the mechanism of platelet activation can be elucidated through the continuous study of platelet function changes in patients with thrombosis, in order to develop new sensitive and specific diagnostic indicators for early postpartum deep venous thrombosis. In future research, we aim to analyze the changes of platelet aggregation activity and PI3K/AKt expression before and after the clinical intervention, to further clarify the predictive value of platelet aggregation activity in postpartum deep venous thrombosis and its mechanism.

In summary, the results of this study revealed that platelet aggregation activity increased in postpartum deep vein thrombosis patients, it has a certain predictive value for the occurrence of postpartum deep venous thrombosis. Furthermore, platelet PI3K expression and AKt phosphorylation levels increased, suggesting that the PI3K/AKt signaling pathway may be one of the mechanisms of platelet aggregation. This further elucidates the changes in postpartum deep vein thrombosis patients and its mechanism, and provide a theoretical basis for the early diagnosis of deep vein thrombosis.

\section{Acknowledgements}

Not applicable.

\section{Funding}

No funding was received.

\section{Availability of data and materials}

The datasets used and/or analyzed during the current study are available from the corresponding author on reasonable request.

\section{Authors' contributions}

MS drafted the manuscript and collected the blood samples. CL helped with platelet aggregation rate. NZ and KM detected platelet activity indicators. ZZ detected PI3K and AKt phosphorylation levels. All authors read and approved the final manuscript.

\section{Ethics approval and consent to participate}

The study was approved by the Ethics Committee of Beijing Chaoyang Hospital (Beijing, China). Written informed consents were signed by the patients and/or guardians.

\section{Consent for publication}

Not applicable. 


\section{Competing interests}

The authors declare that they have no competing interests.

\section{References}

1. Parunov LA, Soshitova NP, Ovanesov MV, Panteleev MA and Serebriyskiy II: Epidemiology of venous thromboembolism (VTE) associated with pregnancy. Birth Defects Res C Embryo Today 105: 167-184, 2015.

2. Skuterud Wik H, Flem Jacobsen A and Morten Sandset P: Long-term outcome after pregnancy-related venous thrombosis. Thromb Res 135: 1-4, 2015.

3. Shifrin MM and Widmar SB: Platelet inhibitors. Nurs Clin North Am 51: 29-43, 2016.

4. Kim SD, Lee YJ, Baik JS, Han JY, Lee CG, Heo K, Park YS, Kim JS, Ji HD, Park SI, et al: Baicalein inhibits agonist-and tumor cell-induced platelet aggregation while suppressing pulmonary tumor metastasis via cAMP-mediated VASP phosphorylation along with impaired MAPKs and PI3K-Akt activation. Biochem Pharmacol 92: 251-265, 2014.

5. Min SH and Abrams CS: Membrane grease eases platelet maturation. Blood 126: 1055-1056, 2015.

6. Buller HR, Sohne M and Middeldorp S: Treatment of venous thromboembolism. J Thromb Haemost 3: 1554-1560, 2005.

7. Konkle BA: Diagnosis and management of thrombosis in pregnancy. Birth Defects Res C Embryo Today 105: 185-189,2015.

8. Parent F, Jovan R and Colas des Francs V: Venous thromboembolism during pregnancy. Rev Prat 65: 188-192, 2015 (In French).

9. Chen Y, Wang Y, Xie Z, Ming X, Li Z and Kong Y: A tryptophan derivative TD-26 attenuates thrombus formation by inhibiting both PI3K/Akt signaling and binding of fibrinogen to integrin $\alpha \operatorname{IIb} \beta 3$. Biochem Biophys Res Commun 465: 516-522, 2015.

10. Singhal R, Annarapu GK, Pandey A, Chawla S, Ojha A, Gupta A, Cruz MA, Seth T and Guchhait P: Hemoglobin interaction with GP1b $\alpha$ induces platelet activation and apoptosis: A novel mechanism associated with intravascular hemolysis. Haematologica 100: 1526-1533, 2015.

11. Lopes-Pires ME, Naime AC, Almeida Cardelli NJ, Anjos DJ, Antunes E and Marcondes S: PKC and AKT modulate cGMP/PKG signaling pathway on platelet aggregation in experimental sepsis. PLoS One 10: e0137901, 2015.

12. Clark GD: Platelet-activating factor acetylhydrolase and brain development. Enzymes 38: 37-42, 2015.
13. Kuriyama N, Mizuno T, Yasuike H, Matsuno H, Kawashita E, Tamura A, Ozaki E, Matsui D, Watanabe I, Koyama T, et al: CD62-mediated activation of platelets in cerebral white matter lesions in patients with cognitive decline. Arch Gerontol Geriatr 62: 118-124, 2016.

14. Pósfai É, Marton I, Kotosz B and Borbényi Z: Contribution of cardiovascular risk factors in the thrombotic complications of essential thrombocythaemia: A Hungarian single-institute retrospective analysis. Eur Rev Med Pharmacol Sci 19: 1258-1263, 2015.

15. Signorelli SS, Ferrante M, Gaudio A and Fiore V: Deep vein thrombosis related to environment (Review). Mol Med Rep 15: 3445-3448, 2017.

16. Gerdsen F, Weber M, Langer F, Eifrig B and Lindhoff-Last E: Platelet activation markers in patients with venous thromboembolism without predisposing factors. Pathophysiol Haemost Thromb 34: 1-5, 2005.

17. Malaponte G, Signorelli SS, Bevelacqua V, Polesel J, Taborelli M, Guarneri C, Fenga C, Umezawa K and Libra M: Increased levels of NF-kB-dependent markers in cancer-associated deep venous thrombosis. PLoS One 10: e0132496, 2015.

18. Mu FT, Cranmer SL, Andrews RK and Berndt MC: Functional association of phosphoinositide-3-kinase with platelet glycoprotein Ibalpha, the major ligand-binding subunit of the glycoprotein Ib-IX-V complex. J Thromb Haemost 8: 324-330, 2010.

19. Kim S, Mangin P, Dangelmaier C, Lillian R, Jackson SP, Daniel JL and Kunapuli SP: Role of phosphoinositide 3-kinase beta in glycoprotein VI-mediated Akt activation in platelets. J Biol Chem 284: 33763-33772, 2009.

20. Kim TH, Kim HM, Park SW and Jung YS: Inhibitory effects of yuzu and its components on human platelet aggregation. Biomol Ther (Seoul) 23: 149-155, 2015.

21. Hao HZ, He AD, Wang DC, Yin Z, Zhou YJ, Liu G, Liang ML, Da XW, Yao GQ, Xie W, et al: Antiplatelet activity of loureirin A by attenuating Akt phosphorylation: In vitro studies. Eur J Pharmacol 746: 63-69, 2015.

(C) $\Theta$ This work is licensed under a Creative Commons cc) Attribution-NonCommercial-NoDerivatives 4.0 International (CC BY-NC-ND 4.0) License. 